\title{
The Proposed Cluster and Position Based Fault Tolerant Multicast Protocol in Ad Hoc Networks
}

\author{
Mahboobeh Abdoos \\ Department of Electrical and Computer Engineering \\ Qom Islamic Azad University, Qom, Iran.
}

\begin{abstract}
A location service gives the requested location information to the nodes in the ad hoc networks. It is supposed that there are different clusters in the different levels. Every cluster has a cluster head. The clusters can be logical or physical. The higher level cluster heads have the information of the tables of lower level cluster heads. The information of tables of a cluster head is updated periodically by its lower level cluster heads. The combination of the cluster and position based routing can be used. In this paper the cluster and position based location service is represented, that every node can find the location of its destination node via the location service. Some nodes are selected as the mirror nodes of a cluster head which can act as the cluster head after the failing of cluster head. These nodes have the replicated information of a cluster head. Here a multicast protocol is proposed that a source node can get the location information of the destination nodes via the proposed location service and puts this information into the header of packets and sends them toward the destination nodes. The simulation results show that the proposed Cluster and Position based Multicast protocol (CPMB) has more packets delivery ratio and packets transfer ratio than the Multicast Ad hoc On-Demand Distance Vector multicast protocol (MAODV).
\end{abstract}

Keywords -Ad hoc network; position service; fault tolerant; cluster; protocol.

\section{INTRODUCTION}

An ad hoc network consists of some wireless mobile nodes which route the packets without any infrastructure. The ad hoc network is divided to static and dynamic ad hoc networks. In a static ad hoc network, the location of a node does not change. In the dynamic ad hoc networks the nodes are moving like the mobile and vehicle ad hoc networks. The topology of the mobile ad hoc network is changing. There are two kinds of routing, the first one is the topology based routing and the second one is the location based routing. The topology based routing use the information of links of the network to transfer the packets. They are divided to the table-driven and the demand based routing protocols. The table-driven routing protocols consist of the distance-vector protocols and the link-state protocols. The location based routing protocols eliminate some topology based limitations, by additional information. They need to the location information of nodes in the network. Every node finds its location by GPS.

A source node uses the location service to find the location of a destination node and puts it into the header of the data packets. every node routing decision is based on the location of destination node and the neighbor nodes. The location based routing protocols do not need to create or support the routes. The nodes do not need to the routing tables and the updating packets. There are different location services like the square location service or simple location service. The section 2 surveys the MAODV multicast protocol and the section 3 reviews the cluster based position service and the section 4 represents the proposed location and cluster based fault tolerant location service then the proposed cluster and position based multicast protocol (CPMB) is represented. In continue the simulation results are shown, which show that the proposed multicast protocol has more packet delivery than the AODV. [1], [2], [3], [4], [5], [6]

\section{MAODV PROTOCOL}

The MAODV is a demand based routing protocol that the necessary routes are discovered. The MAODV has the least control and processing overhead and the multi hop capability and saves the topology dynamically and avoids the loops. Because of the limited resources of an ad hoc network, the MAODV tries to minimize the control overhead by limiting the updating packets. To minimize the processing overhead, the MAODV packets are simple so they need a little computation. MAODV uses the sequential numbers to prevent the loops. In MAODV, every node has a routing table which consists of information of the routes. Every entry of the routing table consists of destination IP address and the sequential number and the number of hops toward the destination node and the next hop toward the destination node and the time to live (TTL) field. When the information of a route is entered in the routing table, the TTL field for destination node, will be set based on the RREQ (route request), RREP (route reply) or the hello packets. 
When a node uses a route toward the destination node, the TTL field will be updated. The neighbor hello packets receiver will update the TTL entry of that node. If a route toward a node is not used and any packet is not received through that route, the routing table TTL entry of that node is not updated. The routes which are not used during the time to live (TTL) field of route will be expired. [7], [8], [9], [10], [11], [12]

\section{Cluster Based Position Service}

\section{A. Geographical Forwarding}

In geographical transfer, every node finds its location by GPS and sends its location and speed to its neighbors by periodic hello packets. The content of Hello packet is represented in table 1. The geographical transfer uses the two-hop distance-vector protocol. A hello packet consists of a list of neighbor nodes and their locations. Every node has some routing tables which have the information of the one and two hop neighbor nodes which are updated through the hello packets. Every tuple in a routing table consists of the ID and velocity and location and time stamp of neighbors. Every tuple in the routing table is expired after the predefined time and is deleted from table. When a node needs to send the packets toward the destination node and has the ID and location information of a destination node, it uses the location information and sends the packets to its nearest neighbor node, to the destination node and this algorithm is repeated. Here is a big problem that if a node does not know that which neighbor node is nearest to the destination node, the GPRS (the Greedy Perimeter Stateless Routing) can solve the problem. It is a geographical routing protocol which uses the sub graph. (See Table 1) [13], [14], [15], [16], [17], [18]

TABLE 1. THE FIELDS OF A HELLO PACKET

\begin{tabular}{|c|c|c|}
\hline \multicolumn{3}{|c|}{ Hello Packet } \\
\hline Source ID & Source Location & Source Speed \\
\hline \multicolumn{3}{|c|}{ Neighbor list: ID and Location } \\
\hline & orwarding Pointer & \\
\hline
\end{tabular}

\section{The Proposed Cluster Based Location SERVICE}

The combination of the cluster and position based routing can be used. Suppose that there are different clusters in the different levels. The clusters can be logical or physical. An accidental unique ID is assigned to every node by using a strong hash function. Every cluster has a cluster head. The cluster head of the lowest level is selected by voting of the nodes of that cluster and the higher level cluster head is defined by the voting of its lower level cluster heads. Every cluster head has a table which is named the cluster neighbor table (Table 2) that its tuples are the neighbor cluster heads of the same level. This table is updated by the neighbor cluster heads. A cluster head has the ID and location information of its cluster nodes in a table, which is named the cluster member table (Table3). Unless the lowest level, every cluster node has a table, which is named the cluster location table that its entries are ID and location of the lower level cluster nodes. (Table 4)
Every cluster head has the location of its higher cluster head. When a node enters a cluster, it sends a packet to the cluster head of that cluster. This packet consists of ID and velocity and the location of that node and time stamp of sending the packet. When a node leaves a cluster, it sends a packet to the cluster head of that cluster. That packet consists of the ID and current location and velocity and movement direction of that node and the time stamp. Via the location of node and the location of the neighbor clusters, it is understood that to which cluster, the node will go. During the predefined time, the location table entry of that node has a pointer to the new cluster which the node wants to enter and after the predefined time, that entry is expired. (See Fig. 1) (See Table 1, Table 2, Table 3)

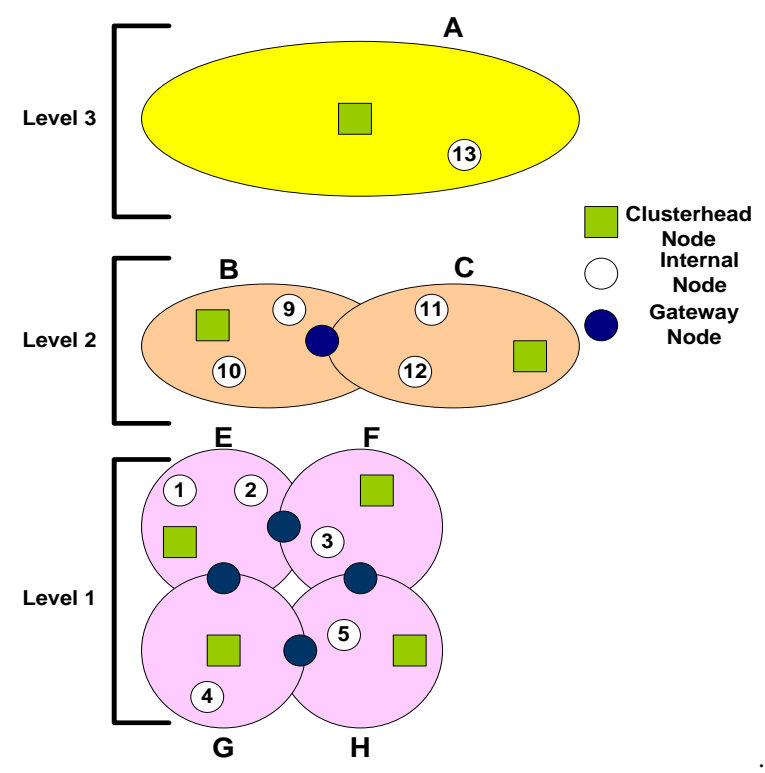

Figure 1.The Structure of Clusters

TABLE 2- THE Cluster NeighBor TABLE

\begin{tabular}{|c|c|}
\hline ID & Location Information \\
\hline 11 & $122^{0} 18^{\prime} 23^{\prime \prime}$ \\
\hline 12 & $110^{0} 17^{\prime} 23^{\prime \prime}$ \\
\hline
\end{tabular}

TABle 3- The Cluster Member TABle

\begin{tabular}{|c|c|}
\hline ID & Location Information \\
\hline 9 & $122^{0} 18^{\prime} 23^{\prime \prime}$ \\
\hline 10 & $130^{0} 18^{\prime} 23^{\prime \prime}$ \\
\hline
\end{tabular}

TABLE 4- THE Cluster LoCATION TABLE

\begin{tabular}{|c|c|}
\hline ID & Location Information \\
\hline 1 & $122^{0} 18^{\prime} 23^{\prime \prime}$ \\
\hline 2 & $115^{0} 20^{\prime} 15^{\prime \prime}$ \\
\hline
\end{tabular}




\begin{tabular}{|c|c|}
\hline 3 & $56^{0} 11^{\prime} 23^{\prime \prime}$ \\
\hline 4 & $122^{0} 1823^{\prime \prime}$ \\
\hline 5 & $47^{0} 3917^{\prime \prime}$ \\
\hline
\end{tabular}

\section{A. Location Request}

When a source node is going to send a data packet toward the destination node, it needs the location of destination node. The source node sends a Location REQuest packet (LREQ) to the cluster head. If the ID and location of destination node exist in the cluster member table or the location table (The entries of location table are ID and location information of its lower level clusters) of the cluster head, then the cluster head puts the location information of destination node into the data packet header and sends it toward the destination node, else the cluster head sends the LREQ to its neighbor cluster heads and the higher level cluster head. At first, It is supposed that the LREQ packet is sent to the neighbor cluster heads and if any response is not received, then the LREQ packet is sent to the higher level cluster head, which is more important node than the neighbor cluster heads. The higher level cluster heads search in their location tables or cluster member tables for the ID and location of the destination node. If this information is found, by one cluster, it puts the location of destination in the Location REPly packet (RREP) header and sends it toward the source node, else at first, the LREQ packet is sent toward the higher level cluster. To which cluster the packet is sent? It depends directly to the distance between the source and destination nodes. If the destination node is so far, the LREQ packet is sent to the higher level and it takes more time. In the hierarchical approach, the time of receiving the LREQ packet, by the destination node directly depends to the distance between the source and destination nodes. If they are near to each other (The destination node is in the cluster of source node or in a cluster near to the source node cluster, the LREQ packet sending time is short. (See Fig. 2)

\section{B. Proposed Fault Tolerant Position Service}

The simple proposed position service is not fault tolerant, because the cluster head is single point of failure and this node may fail under bad situation like the hacking or virus attacking. The information of tables of a cluster head is updated periodically. The cluster head needs to keep the information copy of its cluster member table and neighbor table and location table, and the location of the higher level cluster head in some nodes.

So a cluster head sends the REDundant REQuest packet (REDREQ) to its neighbor nodes in the cluster. If they have the proper situation to keep the redundant information, they reply by the REDundant ACKnowledgement (REDACK), else they send the redundant request packet to their neighbors and reply to the cluster head by REDundant REFuse packet (REDREF). After predefined time, the current cluster head selects some of the nodes which have received the REDACK packet, to keep the redundant information, with attention to their condition. The cluster head sends a copy of table's information to the mirror nodes. The cluster head has the location of the mirror nodes and the mirror nodes update the cluster head by their current location, when a failure occurs and the cluster head fails, the nodes of the cluster will be aware, maybe the cluster head makes them aware, before its failing or the neighbor cluster heads which are aware, make them aware by sending an awareness packet.
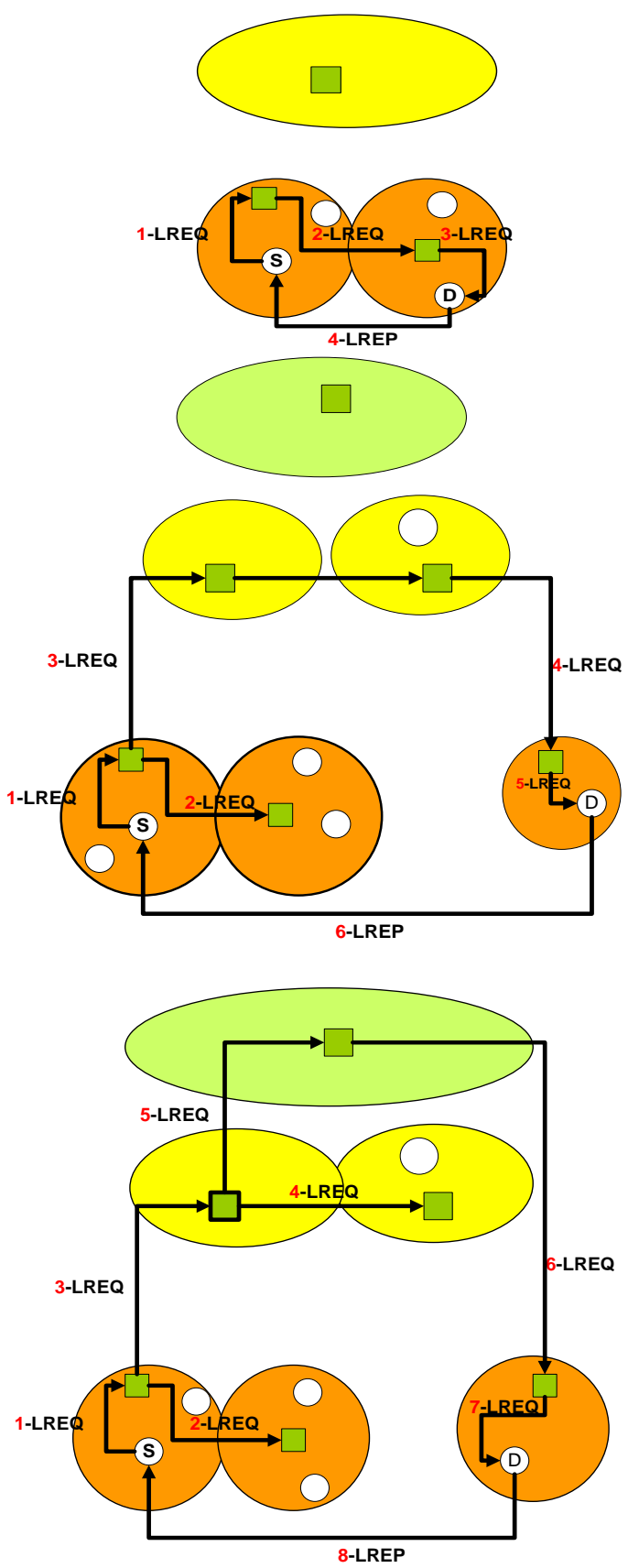

Figure 2. Representation of using the Location Request Packet and (LREQ) the Location Reply Packet (LREP).

In this case the mirror nodes send the awareness packets to the nodes of cluster which contain their ID and current 
location and velocity and the time stamp. The nodes of cluster select the new cluster head. (One of the mirror nodes)

The information of tables of a mirror node is updated by the cluster head. The source node location information receiving, directly depends to the distance between the source and destination nodes. The proposed scheme is fault tolerant. This location service is some-for-some location service. (See Fig. 3)

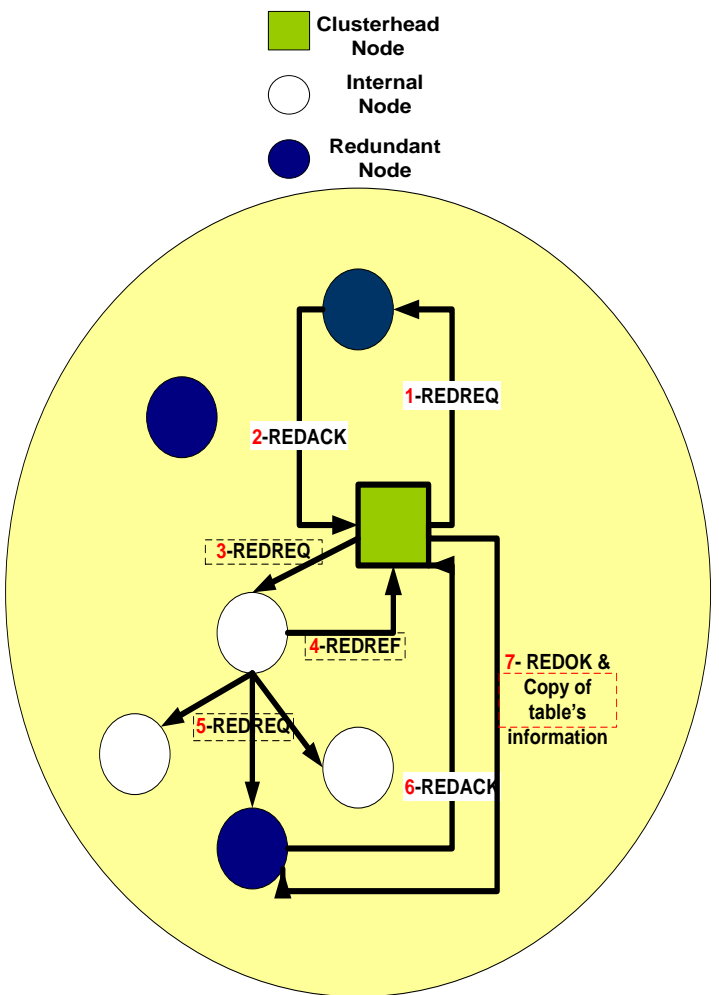

Figure 3. The redundant nodes selection process in a cluster

\section{The Proposed Cluster And Position Based Multicast PROTOCOL(CPMB)}

When a source node wants to send a data packet to some destination nodes, it needs to know the location of destination nodes, so the source node puts the ID of destination nodes in the Location REQuest packet (LREQ) and sends it to the cluster head and the cluster head searches in its cluster member table if there is the location information of the destination nodes, the cluster head puts the location information in the headers of the individual LREQ packets and send them toward the destination nodes. If some location information of destination nodes is not found, the cluster head sends a copy of LREQ packet to the cluster head of the neighbor clusters and the higher level cluster, ....

In every level that the location of destination nodes is found, this information is sent to the source node by the location reply packet. If the source node does not receive the Location REPly packet (LREP), it repeats to send the location request packets until receiving the LREP packet .
If the source node receives the location reply packets from all of the destination nodes, then it puts the location information of destination nodes in the headers of data packets and sends them toward the destination nodes. if after the predefined time, the source node does not receive the acknowledgement (ACK) packets from all of the destination nodes, then it resends the data packets to the destination nodes that have not received the data packets.

\section{SimUlation}

The used simulation tool is NS2. It supports the IEEE 802.11 MAC. The simulation environment consists of 50 wireless mobile nodes that are spread in the $1000 * 1000$ meters during 900 seconds. The radio transfer range is 250 meters. It is supposed that there is free space propagation channel. The group scenarios define that some nodes are receivers of the source node.

A multicast member node, joins a multicast group, with start of the simulation (during first 30 seconds) and will be a member during the simulation. The source node of multicast tree sends (and stops to send) the packets at predefined time interval. (In every one second, four packets are sent and the size of each packet is 512 byte) Here just one multicast group is used. Every mobile node moves accidentally with a predefined speed. Every member of group moves from an accidental location toward an accidental destination location. After stopping the packets sending, another accidental destination is defined. The time of stopping the packets sending, affects the speed of the nodes. The time of stopping the packets sending is set to 0 , because of more dynamism. The speed of the nodes is between $1 \mathrm{~m} / \mathrm{s}$ to $20 \mathrm{~m} / \mathrm{s}$. The used standard metrics are the IETF MANET standards, which are used to evaluate the multicast protocols. The metrics for comparing the MAODV and CPMB are the data packets delivery ratio and the data packets transfer ratio based on the multicast group size and controlling and transferring the data packets based on the delivered data packets and multicast group size. (See Fig. 4, Fig. 5, Fig. 6)

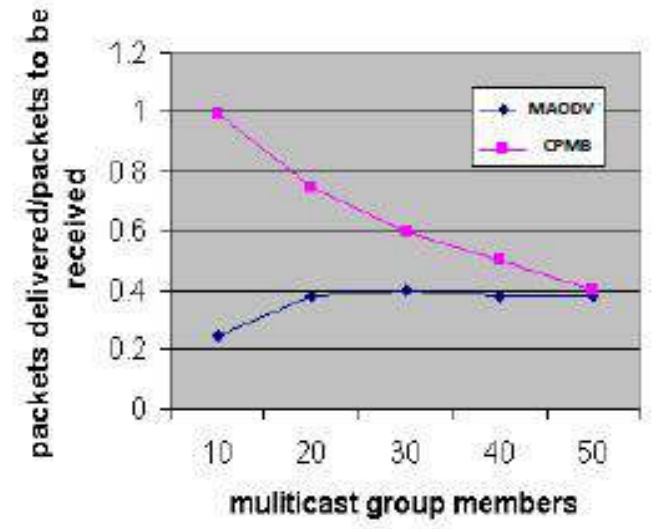

Figure 4 . The data packet delivery ratio, based on the multicast group size 


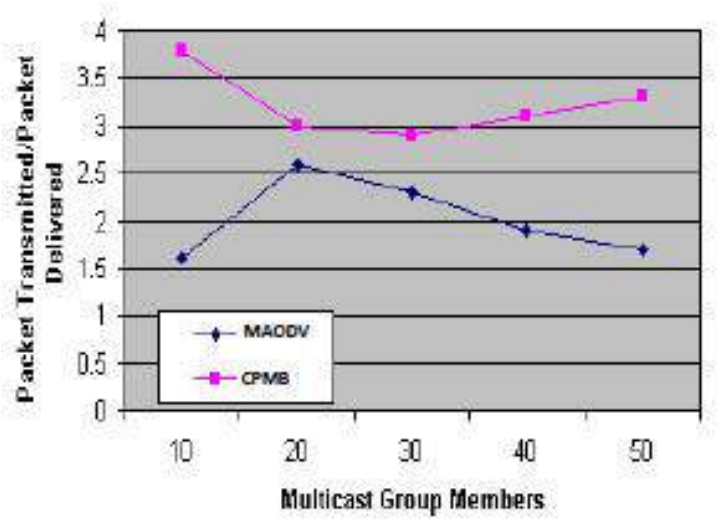

Figure 5. The packets transfer ratio based on the multicast group size

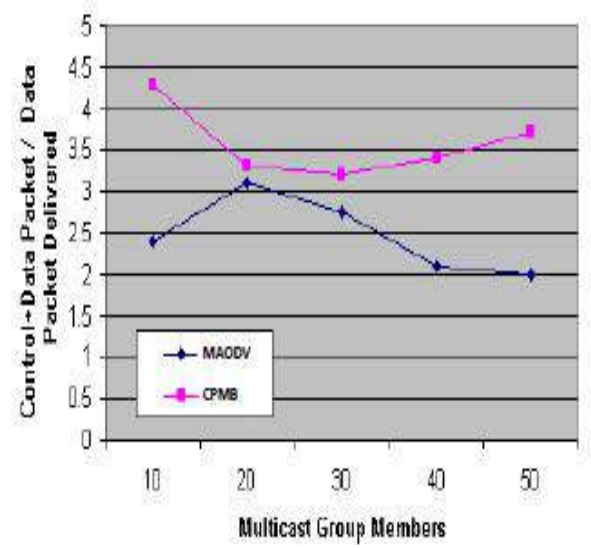

Figure 6. Controlling and transferring the data packets based on the delivered data packets and the multicast group size

\section{CONCLUSION}

In this paper a location service scheme is represented which uses the cluster and position information of nodes. A node can get the location information of another node by this location service. The proposed scheme is scalable and fault tolerant. There are mirror nodes which can act as the cluster head, when the cluster head fails. The information of cluster head tables, are updated periodically.

The cluster head needs to keep the information copy of the cluster member table and the neighbor table and the location table and the location of the higher level cluster at some mirror nodes. a multicast protocol which uses the proposed location service, is explained. The location information of destination nodes is found by the source node and it puts this information in the headers of data packets and sends them toward the destination node. The simulation results show that the proposed multicast protocol has better data packet delivery ratio and better packet transfer ratio than the MAODV multicast protocol.

\section{REFERENCES}

[1] Martin Mauve, Jorg Widmer, Hannes Hartenstein, Nec Europe,Heidelberg, A Survay On Position Based Routing In mobile ad hoc networks, IEEE, 2000.

[2] Slvia Giordano, Ivan Stojmenovic, Ljubica Blazevic, position based routing algorithms for adhoc networks : a TAXONOMY,ICA-DSC-EPFL CH-1015 lausanne (Switzerland), 2002.

[3] Ivan Stojmenovic, location update for efficient routing in adhoc networks, computer science,SITF , university of Ottawa,Ontario KIN 6N5, Canada,2002.

[4] Ivan Stojmenovic, position-based routing in adhoc networks, university of Ottawa and universidead nacional autonoma de mexico,IEEE,2002.

[5] young-Bae Ko, Nitin H . Vaidya,location -aided routing (LAR) in mobile adhoc networks, department of computer .texas A\&M university, college TX 77843-3112,USA,J.C. baltzer AG science publishers, 2000.

[6] Stephen Carter, Alec yasinsac, secure position aided adhoc routing,computer science department Florida state university,Tallahassseee,FL 32306-4530,2002.

[7] V.Sumathy, Dr.P.Nearay anasmy,K.Baskaran,T.Purusothaman, GLS With secure routing in AD-HOnetworks, 0-7803-7651-X/03, IEEE, 2003.

[8] Hannes Frey, Scalable geographic routing algorithms wireless adhoc networks,0890-8044/04, IEEE, 2004.

[9] Martin Mauve, jorg Widmer, Holger Fubler,Thomas Lang, Mobihoc Poster: position-based multicast routing for mobile adhoc networks,Mobile computing and communications review,volume 7,number 3,2003.

[10] Xiaoyan Hong, Kaixin Xu,Mario Gerla, scalable routing protocols for mobile adhoc networks, university of california, los angeles, CA 90095,2002.

[11] david B. johnson, routing in adhoc networks of mobile hosts, computer science department,carnegie mellon university, IEEE, 1994.

[12] Manoj pendey and daniel zappala , the effects of mobility on multicast routing in mobile adhoc networks, computer and information science, university of oregon, 2003.

[13] Kristoffer karlsson, billy ho,adhoc networks: overview, applications and routing issues, chalmers university of technology, 2003.

[14] Megha goel, mark felegyhazi, evaluation of a routing architecture for wireless messaging ad-hoc networks, indian institute of technology delhi(IITD), 2004.

[15] Dragos Niculescu,badri nath,localized positioning in adhoc networks, ELSEVIER, 2003.

[16] Ivan stojmenovic, pedro eduardo villaneuva pena, a scalable quorum based location update scheme for routing in adhoc wireless networks, university of ottawa,2002.

[17] Holger fubler, jorg widmer, michael kasemann,martin mauve, hannes hartenstein,contention -based forwarding for mobile adhoc networks, ELSEVIER, 2003.

[18] Yi cheng, dharma P.agrawal,distributed anonymous secure routing protocol in wireless mobile adhoc networks, university of cincinnati, 2004. 


\section{AUTHOR PROFILE}

Mahboobeh Abdoos received the B.S and M.S degrees in computer engineering from Azad University, Ghazvin, Iran, in 2002 and 2007 respectively. She is now the Ph.D. research student of Islamic Azad university, Qom, Iran. She has taught at Islamic Azad and Payam Nour Universities from 2005 til now. She has been the referee of some conferences. Her current research interest includes position based routing protocols in mobile ad hoc networks, QOS and security based routing protocols in mobile ad hoc networks, cloud computing and data base.

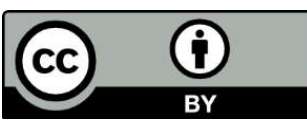

(C) 2016 by the author(s); licensee Empirical Research Press Ltd. United Kingdom. This is an open access article distributed under the terms and conditions of the Creative Commons by Attribution (CC-BY) license. (http://creativecommons.org/licenses/by/4.0/). 